\title{
Tail pinch vs. cervical probing: Opposite effects upon intracranial reinforcement in female rats
}

\author{
R. J. KATZ and M. STEINER \\ Department of Psychiatry, Mental Health Research Institute \\ University of Michigan Medical Center, Ann Arbor, Michigan 48109
}

\begin{abstract}
Tail pinch, that is, the application of mild pressure to the base of a rat's tail for a brief period, has been shown to facilitate a variety of dopamine (DA) dependent motivated behaviors, among them feeding, sexual activity, and intracranial reward (intracranial selfstimulation, ICS). Antelman, Caggiula, and their co-workers have presented a theory of nigro-neostriatal function in which tail pinch and cervical probing, a procedure consisting of the brief application of mild pressure against the wall of the cervix, respectively facilitate and inhibit behavioral activity through opposite effects upon DA cells within the substantia nigra (A9). We tested this model further using A9 implanted female rats responding for ICS. Tail pinch facilitated ICS response rates, while cervical probing reduced them. This is consistent with previously reported behavioral and electrophysiological evidence and with the hypothesized model in which increased and decreased nigrostriatal function serves to activate or suppress behaviors.
\end{abstract}

Tail pinch and cervical probing are two experimental procedures which alter the normal occurrence of behavior in the rat. Tail pinch, that is, the brief application of mild nontraumatic pressure to the base of a rat's tail, increases feeding, sexual behaviors, maternal behaviors, certain classes of stereotyped activity, and self-stimulation behavior (ICS, intracranial self-stimulation) (Antelman \& Caggiula, 1977; Katz \& Roth, 1979). It has been argued, both from behavioral observations and from pharmacological studies, that the behavioral arousal produced by tail pinch is dependent in part upon the extrapyramidal system, particularly ascending dopaminecontaining neurons within this system (Antelman \& Caggiula, 1977). Cervical probing is a second possibly related procedure consisting of the application of brief nontraumatic pressure against the wall of the cervix. In contrast with the tail pinch, cervical probing tends to inhibit behaviors, producing a state of behavioral rigidity and analgesia (e.g., Komisaruk, Ciotalo, \& Latrary, 1976).

A more elaborate and inclusive model of tail pinch and cervical probing elicited behaviors, and their relation to nigro-neostriatal function, was recently proposed by Caggiula, Antelman, Chiodo, and Lineberry (1979). In this theory, tail pinch is considered a prototypic behavioral facilitator acting through the pars compacta of substantia nigra (A9). Cervical probing is regarded as a neurophysiologically and behaviorally opposite influence to the above. This relation-

The technical assistance of Giulio Baldrighi and the statistical assistance of Robert Shea are gratefully acknowledged. ship is supported by unit recordings within the substantia nigra. The present experiments were designed to further test this model, using brain stimulated reinforcement as a dependent variable of interest. We have previously demonstrated brain stimulation in male rats to be responsive to tail pinch (Katz \& Roth, 1979), and this is mediated through DA (Katz, Roth, \& Schmaltz, 1980). The present experiments examined tail pinch and cervical probing upon ICS in female rats.

\section{METHODS}

\section{Subjects}

Fifteen adult ( $\sim 90$ days) female Holtzman rats (Holtzman, Inc., Madison, Wisconsin) were individually maintained with food (Teklad 4\% fat rodent diet S-0836, Madison, Wisconsin) and tap water continuously available. The daily light cycle was between 0545 and $1815 \mathrm{~h}$, and temperature was maintained at $21^{\circ} \mathrm{C}$. Estrous cycles were assessed by daily vaginal lavage, with diestrus defined as the first day in which leucocytes dominated the smear. Fourteen days of apparatus habituation and two complete 4-day cycles were required for inclusion into the sample.

\section{Apparatus}

Detailed descriptions of the apparatus have been published elsewhere (Kata, 1979; Steiner, 1979). The stimulation procedure involved home cage testing using the design of Wolf, DiCara, and Simpson (1973). Cages were modified to house a $14 \times 16 \mathrm{~cm}$ overhead-mounted stainless steel plate which served as both a contact and manipulandum. Upwards displacement of the plate by the rat completed a circuit with the cage floor serving as ground. The plate delivered a $.3-\mathrm{sec}$ pulse of $60-\mathrm{Hz}$ sinusoidal current $(25-75 \mu \mathrm{A})$ through a head-mounted brass brush, which, in turn, connected to a unipolar stimulating electrode. This arrangement provided continuous access to reinforcement without commutators or external leads. Rats may be routinely maintained with continual access to ICS for up to 6 months by this procedure. 


\section{Surgery}

The subjects were anesthetized with $35 \mathrm{mg} / \mathrm{kg}$ sodium pentobarbital (Nembutal, ip) and were stereotactically implanted with unipolar $.025-\mathrm{cm}$-diam nichrome electrodes insulated to the tip. As noted, an external brush was mounted on the skull to permit stimulation delivery. Five stainless steel screws and acrylic dental cement were employed to secure the bushing to the skull. One week postsurgery was routinely permitted for recovery.

\section{Behavioral Procedure}

After recovery, rats were returned to their home cages with the stimulation contingency in effect. Most rats shaped themselves in routine exploration of the box. The remaining rats were shaped by having their heads raised to the plate with a wooden dowel. Three or four sessions of $10 \mathrm{~min}$ duration were sufficient for training. Four-day estrous cycles with the ICS contingency in effect were reestablished over the next 3-4 weeks. It should be noted that by Week 3 greater than $99 \%$ of all ICS responses occurred during the dark period of the day/night cycle.

For Experiment 1, the tail-pinch procedure was administered in accordance with previous descriptions (Katz \& Roth, 1979; Katz et al., 1980). Three sessions of tail-pinch preexposure preceded application of the test stimulus during the week prior to the actual test. This represents a standard procedure in tail pinch experiments, allowing the maximization of goal-directed effects. Although similar effects may be seen upon initial exposure, they are considerably more variable. A standard stimulation consisted of a single 60 -sec exposure to a rubber dam forceps, closed to the first notch, applied 3-4 cm from the base of the tail. Tail pinch was carried out for individual subjects between 1100 and $1400 \mathrm{~h}$, with control periods of undisturbed time samples and mild handling. Subjects were removed from their cages for both handling and tail-pinch procedures. This eliminated the ability to respond during these procedures. Data were recorded for $30 \mathrm{~min}$, and are presented as total responses per 30 -min session following application of the 1-min tail pinch. The testing period was chosen to assure a low baseline level of response.

Cervical probing was carried out manually using a $.64-\mathrm{cm}$-diam stainless steel rod. The rod was inserted until cervical resistance was encountered. Sufficient pressure for continued contact with the cervical wall was maintained for $5 \mathrm{~min}$. Rod insertion and continued contact pressure were effected manually. The pressure of this procedure was sufficient to induce pseudopregnancy in all rats, as measured both by lavage and by loss of otherwise present estrus-related ICS patterning (see Experiment 2 results). Probing was carried out within $30 \mathrm{~min}$ of light offset, with control conditions of an undisturbed time sample and mild handling in which all aspects of the probing procedure were replicated except actual rod insertion. Overnight $(11.5 \mathrm{~h})$ ICS scores served as the dependent variable, and all scores represent total responses per $11.5 \mathrm{~h}$ following the $5 \mathrm{~min}$ of probing or of control procedures. As in the tail-pinch procedure, removal from the cage and opportunity to stimulate was necessary for the application of experimental procedures. This period was used to assure a high baseline of response. For both procedures, tests were carried out once per estrous cycle, repeated across two cycles. Care was taken to test different subjects under all possible estrous conditons.

Statistical analysis was by repeated measures analysis of variance, with data presented as means and standard errors (Dixon \& Massey, 1969).

\section{EXPERIMENT 1}

The first experiment was designed to replicate previous findings upon tail-pinch facilitation of ICS. Previous studies had used male rats (Katz \& Roth, 1979; Katz et al., 1980). The present study extended
Table 1

Tail Pinch Facilitation of Intracranial

Self-Stimulation in Female Rats

\begin{tabular}{lrr}
\hline & \multicolumn{2}{c}{ Score } \\
\cline { 2 - 3 } Group & Mean & \multicolumn{1}{c}{ SE } \\
\hline Control (Basal) & 0 & 0 \\
Control (Handled) & 75 & 50 \\
Experimental (Tail Pinch) & 331 & 70 \\
\hline
\end{tabular}

Note-Numbers represent total ICS responses for the 30-min session immediately following tail pinch. $N=8$.

the analysis to female rats of a novel strain. In further contrast with previous experiments, electrodes were aimed at the substantia nigra.

Materials and methods used were as described above.

Tail pinch facilitated ICS responding for eight of eight rats. This facilitation occurred at a statistically reliable level in comparison with either control condition $[\mathrm{F}(2,15)=5.5, \mathrm{p}<.05]$. These findings are presented in Table 1.

The first experiment replicated two previous findings involving male rats. While this is not surprising, the use of both a new site and of female rats of a novel strain suggests that the facilitation effect may be regarded as fairly general. Based upon the model of Caggiula and Antelman, it is predicted that cervical probing should have an opposite, rate-inhibitory effect. To test this, an additional group of female rats were probed immediately prior to light offset, a period of normally high ICS responding.

\section{EXPERIMENT 2}

The present experiment examined the effects of cervical probing upon intracranial reinforcement. Probing has previously been shown to produce analgesia and immobility (Komisaruk et al., 1976). No studies to date have examined effects upon intracranial reward.

\section{Methods}

The materials and procedures were identical with previous descriptions. At the close of this experiment, a total of six rats were chosen at random from both experiments for histological examination by the method of Hosko (1975).

\section{Results}

Figure 1 presents representative individual records of self-stimulating rats, showing normal patterning, cervical probing reductions in behavior, and the subsequent induction of pseudopregnancy as determined by a chronic reduction in normal estrus-related ICS patterning. It should be emphasized that both estrus and pseudopregnancy were further verified by lavage, as indicated above. 


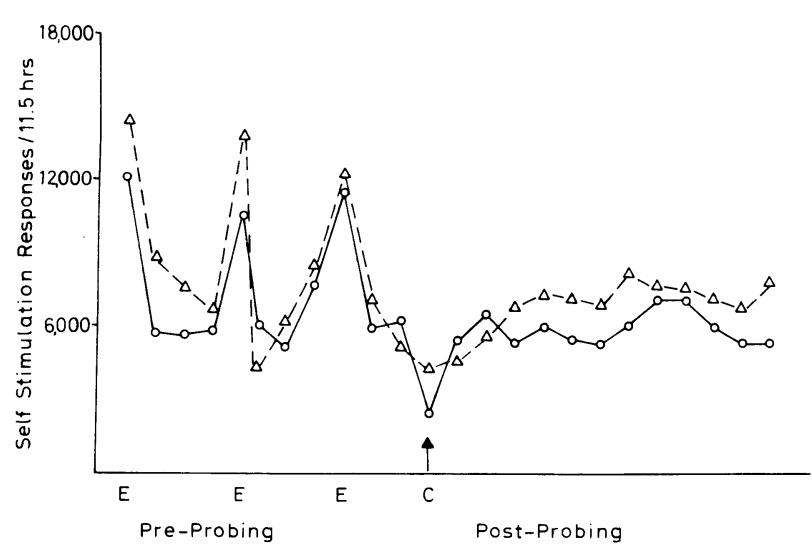

Figure 1. Representative daily (i.e., $11.5 \mathrm{~h}$ overnight) records of two adult female rats chronically self-administering brain stimulation. The figure displays normal estrus patterning (left-hand portion), cervical probing induced reductions (central part), and subsequent behavioral evidence of a loss of estrus patterning (i.e., pseudopregnancy). See text for further descriptions of behavioral procedures. $C=$ cervical probing; $E=$ estrus as defined by lavage (NB, probing was performed a second time only after the reestablishment of regular estrous cycles).

Inhibition of responding due to cervical probing was found in six of seven rats $(p<.05$ by binomial expansion). This was reflected in a significant difference across groups (see Table 2), with the experimental condition's average score being reduced by approximately half. This finding was significant $[\mathrm{F}(2,12)$ $=4.9 ; \mathrm{p}<.05]$. Histology indicated that all placements were in pars compacta of substantia nigra. ${ }^{1}$

\section{DISCUSSION}

While previous reports have emphasized a profound immobility after cervical probing, rats in the present design performed on the average of 10,000 barpresses per night. This represents a significant reduction from normal activity, although it is a high level of activity itself. One reason for this may rest with the use of a testing session of sufficient (i.e., $11.5 \mathrm{~h}$ ) length to allow both an immediate effect and partial recovery over time.

By this explanation, cervical probing may have produced an immediate state of poststimulation immobility, which gradually was superseded by normal

Table 2

Inhibition of Intracranial Reinforcement in Female Rats by Cervical Probing

\begin{tabular}{lcc}
\hline \multirow{2}{*}{ Group } & \multicolumn{2}{c}{ Score } \\
\cline { 2 - 3 } \multicolumn{1}{c}{ Control (Basal) } & Mean & SE \\
Control (Handled) & 16,980 & 2,160 \\
Experimental & 15,620 & 3,920 \\
\hline
\end{tabular}

Note-Numbers represent total ICS response per overnight (i.e., 11.5-h session following cervical probing). $N=7$. rates of responding. Reduction of the overnight average may reflect the interaction of both factors in producing a net lower score. Should this be the case, it might explain the apparently reduced statistical reliability of handled control vs. experimental rats. Shorter periods may be more exclusively sensitive to immobility effects.

The present findings support and extend demonstrations by Komisaruk and others of profound motivational changes after cervical probing (Komisaruk et al., 1976). They also support the model of Caggiula and Antelman, suggesting that their neurophysiological findings have behavioral concomitants. It must, however, be cautioned that the present experiments examined only a single nucleus, albeit the one considered most critical to the theory. Since only one site was employed, the generality of these findings to other brain systems is not established.

\section{REFERENCES}

Antelman, S. M., \& Caggiula, A. R. Tails of stress related behavior: A neuropharmacological model. In I. Hanin \& E. Usdin (Eds.), Animal models in psychiatry and neurology. New York: Pergamon, 1977.

Caggivla, A. R., Antelman, S. M., Chiodo, L. A., \& Lineberry, C. G. Brain dopamine and sexual behavior: Psychopharmacological and electrophysiological evidence for an antagonism between active and passive components. In E. Usdin, I. J. Kopin, \& J. Barchas (Eds.), Catecholamines, basic and clinical frontiers (Vol. 2). New York: Pergamon, 1979.

Dixon, W. J., \& MASSEy, F. J. Introduction to statistical analysis. New York: McGraw-Hill, 1969.

Hоsко, M. J. Technique for rapid permanent documentation of intracerebral electrode sites. Physiology \& Behavior, 1975, 14, 367-368.

KATZ, R. J. The temporal structure of reinforcement: An analysis of brain stimulated reward. Behavioral and Neural Biology, 1979, 26, 416-430.

KATZ, R. J., \& Roth, K. A. Tail pinch induced stress-arousal facilitates brain stimulation reward. Physiology \& Behavior, 1979, 22, 193-194.

Katz, R. J., Roth, K. A., \& Schmaltz, K. E. Tail pinch facilitation of self stimulation in the rat-Dependence upon dopamine and independence of opiates. Pharmacology, Biochemistry and Behavior, 1980, 12, 389-391.

Komisaruk, B. R., Ciotalo, V., \& Latrary, M. B. Stimulation of the vaginal cervix is more effective than morphine in suppressing a nociceptive response in rats. in $\mathbf{J}$. J. Bonica \& D. Able-Fresard (Eds.), Advances in pain research. New York: Raven, 1976.

Steiner, M. Motivated behavior and the estrus cycle of the rat. Unpublished doctoral dissertation, University of Michigan, 1979.

Wolf, G., DiCara, L. V., \& Simpson, W. The contact method, a simple technique for electrical self stimulation without external leads. Physiology \& Behavior, 1973, 11, 721-723.

\section{NOTE}

1. A more complete pictorial histology has been published. See Steiner (1979).

(Received for publication January 28, 1980: revision accepted April 15. 1980.) 